\title{
Analysing exponential random graph (p-star) models with missing data using Bayesian data augmentation
}

\author{
Johan H. Koskinen ${ }^{\mathrm{a}, \mathrm{b}, *}$, Garry L. Robins ${ }^{\mathrm{b}}$, Philippa E. Pattison ${ }^{\mathrm{b}}$ \\ ${ }^{\text {a }}$ ReMiSS, Department of Politics and International Relations, University of Oxford, Manor Road, Oxford OX1 3UQ UK \\ ${ }^{\mathrm{b}}$ Department of Psychology, University of Melbourne, Parkville, 3010 Vic., Australia
}

\section{A R T I C L E I N F O}

\section{Article history:}

Received 18 April 2009

Received in revised form

21 September 2009

Accepted 22 September 2009

\section{Keywords:}

Missing data

Data augmentation

Exponential random graph models

Auxiliary variable

Social network analysis

\begin{abstract}
A B S T R A C T
Missing data are often problematic in social network analysis since what is missing may potentially alter the conclusions about what we have observed as tie-variables need to be interpreted in relation to their local neighbourhood and the global structure. Some ad hoc methods for dealing with missing data in social networks have been proposed but here we consider a model-based approach. We discuss various aspects of fitting exponential family random graph (or p-star) models (ERGMs) to networks with missing data and present a Bayesian data augmentation algorithm for the purpose of estimation. This involves drawing from the full conditional posterior distribution of the parameters, something which is made possible by recently developed algorithms. With ERGMs already having complicated interdependencies, it is particularly important to provide inference that adequately describes the uncertainty, something that the Bayesian approach provides. To the extent that we wish to explore the missing parts of the network, the posterior predictive distributions, immediately available at the termination of the algorithm, are at our disposal, which allows us to explore the distribution of what is missing unconditionally on any particular parameter values. Some important features of treating missing data and of the implementation of the algorithm are illustrated using a well-known collaboration network and a variety of missing data scenarios.
\end{abstract}

(C) 2009 Elsevier B.V. All rights reserved.

\footnotetext{
Previous versions were presented at the 2007 Sunbelt XXVII, International Sunbelt Social Network Conference, Greece; and at the 2007 8th Asia-Pacific Complex Systems Conference, Australia; and the authors have benefited from numerous comments and suggestions by participants. This research has been supported by funding from the Australian Defence Science and Technology Organisation.

* Corresponding author at: ReMiSS, Department of Politics and International Relations, University of Oxford, Manor Road, Oxford OX1 3UQ, UK. Tel.: +44 018652785 24; fax: +44 01865278725.

E-mail address: johan.koskinen@nuffield.ox.ac.uk (J.H. Koskinen).
} 


\section{Introduction}

In the social network framework, social interaction is typically interpreted with respect to a network defined by a fixed set of actors [58]. Thus, central notions of social interaction are intimately related to the definition of the boundary of the network [35]. While determining a meaningful definition of what constitutes the network (not only the set of actors but also relations, etc.) is ultimately a theoretical question, the general issue of boundary definition also has practical consequences for empirical investigation. As pointed out by Stork and Richards [53], ignoring missing data (more particularly in their case non-respondents) can amount to redefining the boundary. The theoretical and practical implications of this are further discussed in [28] and [5].

Thus far relatively scant attention has been paid to the treatment of missing data. Stork and Richards [53] discussed and reviewed the problem and proposed an ad hoc scheme for complementing data in the face of non-respondents. This had the advantage that the network may be analysed on its intended scale but this advantage comes with several disadvantages [28,46]. Kossinets [28] reviewed extensively the topic of missing data and investigated the performance of some common graph indices under different missing data scenarios. Other accounts of the effect on indices of missing data were given by Costenbader and Valente [9] and [27]. Huisman [27] translated several imputation strategies that are common in the statistical literature to the context of social network data but it appears that the answer as to which imputation scheme is most effective is inconclusive. Hence, researchers commonly acknowledge the dangers of ignoring missing data but practical advice appears difficult to give in the general case. A reason for this is that while features of the observed part of the graph are influenced by the unknown values, it is not clear how the knowledge about the observed features of the graph may inform us about the unknown values. This is the case, at least, if we do not have a model for how the observed relates to the unobserved available (a notable exception is Hoff [21], who provides a model-based approach to prediction of missing data).

The family of exponential random graph (or p-star) models (ERGM) has been proposed as a family of models that is able to model social network data, with the type of complex interdependencies this typically entails $[23,13,11,59,42,45,52,26]$. Representing statistical models for networks, the ERGMs have the potential for allowing us to use the model and observed data to inform us about what is missing. ERGMs in the context of missing data have earlier been treated by Robins, Pattison, and Woolcock [46], whereby non-respondents were treated as a particular category of actors. Their approach consequently allowed estimation of the model only under very specific assumptions. Recently Handcock and Gile $[17,20,19]$ outlined an approach to maximum likelihood inference for ERGMs with missing data under the assumption of data missing at random. A particular merit of these papers is that they highlight the connections between ERGMs with missing data and inference for various sampling designs; the latter is a field in which a considerable amount of statistical research has been undertaken (see e.g. [55]).

The contribution of this article is as follows. We present an approach for Bayesian analysis of ERGMs when data are partially missing (missing at random) for a fixed and known set of nodes. In the course of doing this, we propose a procedure for finding the approximate modal point of the posterior and the information matrix for curved ERGMs with missing data. We furthermore introduce link prediction for social networks using ERGMs. As degeneracy has been shown to be a problem in maximum likelihood analysis of ERGMs $[8,51,18,52]$, we present some results regarding the existence of the posterior distribution for ERGMs and how this translates to the case of missing data. For a commonly used specification of the social circuit ERGM [52], we also give a series of easy to check criteria for the existence of the posterior. To illustrate the implementation of the missing data algorithm, we give in-depth examples of the effect on inference of missing data in a few illustrative cases, with nonrespondents and snowball-sampled data, as well as brief comparison of predictions with a latent variable model for the case of dyads missing at random.

A Bayesian approach has the benefit over a non-Bayesian approach in that uncertainty is more clearly defined and handled: we want to take the added uncertainty due to unknown data into account when interpreting the model in terms of its parameters and the predictive, goodness of fit, distributions. Additionally, though not explicitly treated here, a Bayesian approach allows for 
incorporating prior information, something that may prove essential when data are scarce but decisions have to be made.

The rest of the paper is structured as follows. In Section 2, the model is defined along with some necessary definitions and notation for missing data. Some analytical bounds on the degree to which data may be degraded are presented. Section 3 presents the inference scheme with explanations of the principal inferential goals. A well-known data set is used to illustrate various aspects of fitting ERGMs to data with missing observations (Section 4), followed by a concluding section.

\section{The model and missing data structure}

Throughout we assume that we are interested in modelling graphs or digraphs on a fixed set $V=\{1, \ldots, n\}$ of vertices (the case of a partially unknown set of vertices is further discussed in [32]). To this end, we define the model for binary adjacency matrices $x$ that take values in $X$. The proposed methods do not vary greatly for valued graphs, multigraphs, bipartite graphs or other graph objects for which ERGMs have been defined but for the purpose of making the presentation clear, the approach is presented assuming an undirected, univariate network.

Let $\mathcal{N}=\left(\begin{array}{l}v \\ 2\end{array}\right)$ denote the index set of the non-redundant elements of $x=\left(x_{e}: e \in \mathcal{N}\right)$, so that for the stochastic edge set $E \subseteq \mathcal{N}, x_{e}=1\{e \in E\}$ is the usual tie variable, and $\mathcal{X}=\{0,1\}^{\mathcal{N}}$. When it is clear from the context, $x$ denotes the conventional square, symmetric adjacency matrix. Define the probability mass function (pmf) by

$$
p(x \mid \theta)=\exp \{q(x ; \theta)-\psi(\theta)\},
$$

where $q$ is a real-valued function of $x$, a $p \times 1$ vector of parameters $\theta \in \Theta \subseteq \mathbb{R}^{p}$, and possibly some fixed set of covariates, and where $\psi(\theta)=\log \sum_{x \in X} \exp \{q(x ; \theta)\}$ is a normalising constant ensuring that $p$ sums to one over $\mathcal{X}$. For exponential family random graph distributions $q(x ; \theta)$ may be expressed in terms of $\theta^{\mathrm{T}} z(x)$ the inner product of a natural parameter and a $p \times 1$ vector valued function $z(x)$. For curved exponential family random graph models [24,26], there exists a function $\eta: \theta \rightarrow \mathbb{R}^{p^{\prime}}$, for $p^{\prime}>p$, such that $q(x ; \theta)=\eta(\theta)^{\mathrm{T}} z(x)$, for some function $z$ of the adjacency matrix $x$ and covariates.

In ERGMs, $z$ may include the statistics: degree of the graph, $\sum x_{i+}$, where $x_{i+}=\sum_{j} x_{i j}$ is the degree of node $i$; stars of order $k, \sum\left(\begin{array}{c}x_{i+} \\ k\end{array}\right)$; the number of triangles, $\sum_{1 \leq i<j<h \leq h} x_{i j} x_{i h} x_{j h}$, etc. For a covariate $a_{i}$, we may for example include the main effect, $\sum x_{i j}\left(a_{i}+a_{j}\right)$, and the homophily effect, $\sum_{i<j} x_{i j} \mathbf{1}\left\{a_{i}=a_{j}\right\}$ (for sufficient statistics see [44]).

There are many forms that missingness can take in social network analysis and while most real applications are likely to have elements of several forms, we focus here on the case that satisfies the set of assumptions that [32] refer to as "the standard model". Firstly, we assume the particular case where we unambiguously may determine whether a tie-variable $x_{e}$ is observed or not. Note that while this covers the case of non-respondents for directed networks, it might not automatically cover the case of non-respondents in undirected networks or other instances where the observations are laden with measurement error (such as in the case of cognitive social structures [34]). The standard model and deviations from it are further described in [32] in the context of common social network issues.

Let $\xi=\left(\xi_{e}: e \in \mathcal{N}\right)$ be a collection of indicator variables such that $\xi_{e}$ is equal to one if the tievariable $x_{e}$ is observed, and zero otherwise. For a given $\xi$, we shall write $x=(u, v)$ using the notational convention that $u$ denotes the collection of tie-variables that are observed and $v$ denotes the collection of tie-variables that are unobserved. Sometimes it shall be convenient to refer to the missing elements using the index set $M=M(\xi)=\left\{e \in \mathcal{N}: \xi_{e}=1\right\}$, and the complement $M^{c}=\mathcal{N} \backslash M$, and the conditional range-space $\mathcal{X}_{M}(u)=\left\{x \in \mathcal{X}: x_{e}=u_{e}, e \in M\right\}$. Central to the proposed methodology is that the pmf of $\xi$ has the property

$$
p(\xi \mid u, v)=p(\xi \mid u),
$$

where we have suppressed the notational dependence of the pmf on some parameter distinct from $\theta$. That is, tie-variables are missing at random (MAR) in the sense of [48] and consequently, with prior 
distribution $\pi(\theta)$, for the posterior we have

$$
\begin{aligned}
\pi(\theta \mid u, \xi) & \propto \pi(\theta) \sum_{v} p(\xi \mid \theta, u, v) p(u, v \mid \theta) \\
& =\pi(\theta) p(\xi \mid u) \sum_{v} p(u, v \mid \theta) \\
& \propto \pi(\theta) \sum_{v} p(u, v \mid \theta) .
\end{aligned}
$$

Henceforth, summation over $v$ is taken to mean summation over elements in $\mathcal{X}_{M}(u)$. Consequently, if tie-variables are missing at random then we do not have to take into account what causes observations to be missing when doing inference for the parameters. The missing data mechanism can to all intents and purposes be ignored (hence the term "ignorable" $[20,19]$; note that "ignorable" in addition to MAR requires "distinct" parameters, see [48] p. 587). Thompson and Frank [55] showed that most linktracing designs are missing at random, and [20] demonstrates this for the particular case of ERGMs as well as gives several examples of sampling designs that satisfy this criterion.

A simple form of missing data mechanism is when individual tie variables are missing independently with a probability that does not depend on their value. That individual tie-variables are missing is what Huisman [27] called item non-response. If the missing data are accumulated within actors, assuming all ties pertaining to an actor to be missing, we have what Huisman [27] calls unit non-response. This roughly translates to respondent non-response (for self-reported ties) and may not in itself violate MAR. For purposes of inference, item and unit non-response are computationally equivalent provided that MAR holds true. For directed networks where actors report on their outgoing ties, $\xi_{i j}=0$ if $i$ is a non-respondent and 1, otherwise, which under suitable assumptions is ignorable (for a few technical definitions see [20]). In Huisman's [27] terminology, unit and item nonresponse both encompass the case when information may be missing for exogenous covariates as well as tie-variables, which seems a realistic scenario, but which is left out of the present presentation.

In a snowball sampling design (see [55]), what is observed depends on an initial sampling mechanism as well as the realised graph, $x$. Despite this, as Handcock and Gile [20] observe, the sampling mechanism is ignorable. If the initial sample is denoted by $s_{0} \subset V$, and iteratively, a node $j \in s_{k}$, where $s_{k}$ is wave $k$, if there exists an $i \in \cup_{r=0}^{k-1} s_{r} \backslash\{j\}, x_{i j}=1$. For two waves, the inclusion variable $\xi_{i j}$ thus indicates whether $i \in s_{0} \cup s_{1}$ or $j \in s_{0} \cup s_{1}$.

It is tempting to perform inference only with what one has observed. The function $L(\theta ; u)=$ $\sum_{v} p(u, v \mid \theta)$ is called the face likelihood and is a function of $\theta$ given $u$ that does not depend on any particular $v$. To say that ignorability implies that we may ignore the sampling mechanism allows us to ignore any particular $v$ but does not translate into saying that we may ignore the fact that data are missing. The case when we ignore the fact that data are missing we refer to as available case analysis (cf. [38]), and corresponds to assuming the likelihood to be $p(u \mid \theta)=\exp \{q(u ; \theta)-\psi(\theta)\}$ (whenever $q(u ; \theta)$ is defined, which it is not for example in the case of unit non-response). While available case analysis may lead to serious bias in non-network data [38,32] discuss how this may be especially detrimental in social network analysis. In particular, one can use the graphical modelling framework [36] to show that removing tie-variables means adding ties between tie-variables in the dependence graph, from which the non-zero interactions (i.e. the interactions that correspond to the configurations from which the sufficient statistics are derived see [11]) are determined. Removing missing data thus corresponds to adding non-zero interactions, increasing the complexity of the configurations we need to include in $z(x)$. Thus, if the distribution for a random graph follows an ERGM, the induced distributions for its subgraphs do not follow an ERGM with the same statistics [32].

\subsection{Limits on degradation}

For estimability we may distinguish between the question of whether the observed data provide practically useful information about the model and whether the posterior distribution exists at all. If a proper prior distribution is used for $\theta$ then the posterior will always exist but in extreme cases 
this posterior may be completely determined by the prior distribution if the data do not add any information. An 'improper' or 'uniform' prior is throughout understood to mean a uniform prior for $\theta$ on $R^{p}$. As pointed out by a reviewer, there may be other 'improper' priors for which these results do not hold.

Handcock [18] provides a non-Bayesian version of Lemma 1 for completely observed data that draws on general results for exponential family distributions [3], but Proposition 2 also applies to the existence of the missing data MLE. In the following, we assume that we have a model $p(x \mid \theta)=$ $\exp \left\{\theta^{\mathrm{T}} z(x)-\psi(\theta)\right\}$, the image of $\mathcal{X}$ under $z$ is denoted by $\mathcal{Z}$, and $C$ is the relative interior of the convex hull on $\mathcal{Z}$. Recall that the posterior with improper prior is proper if $\exp \left\{\theta^{\mathrm{T}} z(x)-\psi(\theta)\right\}$ is integrable.

Lemma 1. For an observation $x$, the posterior is proper if and only if $z(x) \in C$.

Proof. A proof follows directly from the proof of Theorem 1 in [10] upon observing that $\exp \left\{\theta^{\mathrm{T}} z(x)-\right.$ $\psi(\theta)\}$ is functionally equivalent to their conjugate prior for the special case $n_{0}=1$.

Proposition 2. For observed data $u$, with missing data assumptions as above, the posterior distribution exists if and only if $z(x) \in C$ for every potential realisation $x=(u, v)$.

Proof. By definition, the posterior proportional to the face likelihood is integrable if

$$
\int \sum_{v} p(u, v \mid \theta) \mathrm{d} \theta=\sum_{v} \int p(u, v \mid \theta) \mathrm{d} \theta<\infty .
$$

From the positivity of the pmf and that the sum is finite, for the posterior given $u$ to exist we must have $\int p(u, v \mid \theta) \mathrm{d} \theta<\infty$ for all $v$. Hence, the posteriors given all the potential observations $x=(u, v)$ must exist, and thus by Lemma $1 z(u, v) \in C$ for all $v$. The converse is likewise easy to establish using the positivity of the pmf and that the sum is finite.

In other words, if there is even one "extreme" graph among the possible graphs that we could have observed, then the posterior does not exist (nor does the MLE).

For a curved exponential model where fixing one parameter $\theta_{p}$ and considering this a known constant, defines an ERGM, we call this ERGM an ERGM projection. It is easy to see that when the ERGM projection given a particular $\theta_{p}$ results in the posterior not being proper for partially observed data $u$, then the posterior does not exist for the full curved ERGM. For models that include "social circuit " statistics [52], there exists a simple ERGM projection, and we may then easily check to see if the model is degenerate.

To emphasize the practical implications of Proposition 2, the following observation is helpful.

Observation 3. For observed data $u$, any model that has a model nested within it that is inferentially degenerate in the sense of Proposition 2 is also inferentially degenerate.

Even though this result is self-evident from the definition of the convex hull (in the case of no missing data, from which Proposition 2 follows), it is nonetheless important since it implies that we can never fit a model if it has an inferentially degenerate model nested in it and that we can never alleviate a degeneracy issue for a model by merely including extra parameters. The practical implications of this may be that if we find one model that is degenerate then we need not fit any model in which it is nested and it does not matter in what order we fit the parameters of nested models. A concrete example of the consequence of Observation 3 is that we cannot fit any model containing a statistic for the number of isolated nodes if we have missing actors.

\subsubsection{The edges and alternating $k$-triangle model}

What the analytical results imply depends crucially on whether the model allows us to ascertain whether the posterior exists according to Proposition 2. The degeneracy problems are prevalent in practice for homogeneous Markov models than for the so-called "social circuit" ERGMs [52] (for an indepth treatment of degeneracy for Markov models, see [18]). Surprisingly, for the model that contains 
only the number of edges and the alternating $k$-triangle statistic [52] as statistics, establishing whether the posterior exists according to Proposition 2 is simple. The alternating $k$-triangle statistic is given by $A K T(x ; \lambda)=\lambda \sum_{i<j} x_{i j}\left\{1-(1-1 / \lambda)^{S_{2 i j}}\right\}$, where $S_{2 i j}=\sum_{h} x_{i h} x_{h j}$. We offer the following without a detailed proof.

Proposition 4. For observed data $u$, and a model with $z(x)=\left(\sum_{e \in \mathcal{N}} x_{e}, A K T(x ; \lambda)\right)^{\mathrm{T}}$, and $\sum_{e \in M} x_{e}+$ $\left|M^{c}\right| \leq\left\lfloor\frac{n}{2}\right\rfloor\left\lceil\frac{n}{2}\right\rceil$, the posterior exists if the subgraph induced by the edges of $u$ has at least one triangle.

Proof. By Proposition 2 we need to show that $z(u, v) \in C$ for all $v$. If the subgraph induced by the edges of $u$ has at least one triangle, $x=(u, v)$ must have at least one triangle for all $v$ while $\sum_{e \in M} x_{e}+\sum_{e \in M^{c}} x_{e}+\leq\left\lfloor\frac{n}{2}\right\rfloor\left\lceil\frac{n}{2}\right\rceil$, upon which the proof of Proposition 2 in [32] follows.

The remarkable thing is that the model is inferentially non-degenerate if the observation has any triangles as long as it does not have all triangles! This is a clear departure from some Markov models, say, e.g. the edges and two-path model, which has plenty of graphs on the relative boundary. Additionally, this is valid for all values of $\lambda$ and thus the posteriors may exist even for the curved exponential family model.

\section{The algorithm}

The standard approach of data augmentation [54] consists of alternating between drawing from the full conditional posteriors $\pi(\theta \mid u, v)$ and $\pi(v \mid u, \theta)$ that produces a sequence $\left(\theta^{(g)}, v^{(q)}\right)$ of variates from the joint posterior distribution $\pi(\theta, v \mid x)$.

\subsection{Drawing from the full conditional posterior of the parameters}

When drawing from the posterior $\pi(\theta \mid x)$, one may usually use the fact that most MCMC samplers only require that the posterior is known up to a constant of proportionality. The only part of $\pi(\theta \mid x)$ we need to be able to evaluate is $p(x \mid \theta) \pi(\theta)$. In the case of ERGMs however, the likelihood $p(x \mid \theta)=$ $\exp \{q(x ; \theta)-\psi(\theta)\}$ contains the function $c(\theta)=\exp \psi(\theta)$ which is analytically intractable. An MCMC scheme for drawing from such distributions was proposed by Møller et al. [40] but proved to work poorly for social network data [29]. For drawing from $\pi(\theta \mid u, v)$ we use the linked importance sampler auxiliary Metropolis-Hastings (LISA) [29]. LISA has proved to work effectively for ERGMs (and so-called social influence models [29]) but there are other recently proposed algorithms [39,2] that may work equally well.

LISA solves the problem of an intractable $c(\theta)$ by defining a Markov chain $\left(\theta^{(g)}, \omega^{(g)}\right)$, for $\omega^{(g)}$ in an extended state space $\Omega \subseteq \prod_{j=1}^{m} X^{K} \times\{1, \ldots, K\} \times\{1, \ldots, K\}$. The variable $\omega$ is made up of $m$ sequences of $K$ Metropolis-Hastings draws, with target distributions $p\left(x \mid \theta_{j}\right)$, linking $\theta_{1}=\zeta$ with $\theta_{1}=\theta$. This defines a distribution $P_{\zeta, \theta}^{B}(\omega)=Q_{\zeta, \theta}^{B}(\omega) / c(\zeta)$ on $\Omega$, with the property that it has a reverse distribution $P_{\theta, \zeta}^{F}(\omega)=Q_{\theta, \zeta}^{F}(\omega) / c(\theta)$, and we may draw from

$$
\pi(\omega, \theta \mid x)=P_{\zeta, \theta}^{B}(\omega) \pi(\theta \mid x) \propto Q_{\zeta, \theta}^{B}(\omega) \frac{\mathrm{e}^{q(x ; \theta)}}{c(\theta)} \pi(\theta),
$$

using Metropolis-Hastings for a particular choice of proposal distributions. For proposal distributions, let a candidate $\theta^{*}$ be proposed from a multivariate normal distribution $N\left(\theta^{(g)}, \Psi\right)$, centred over the previous state, and conditional on the proposed $\theta^{*}$, propose $\omega^{*}$ from the distribution $P_{\theta^{*}, \zeta}^{F}\left(\omega^{*}\right)$. The Hastings acceptance ratio becomes

$$
\begin{aligned}
& \frac{\pi\left(\omega^{*}, \theta^{*} \mid x\right)}{\pi(\omega, \theta \mid x)} \frac{P_{\theta, \zeta}^{F}(\omega) \varphi\left(\theta^{(g)} ; \theta^{*}, \Psi\right)}{P_{\theta^{*}, \zeta}^{F}\left(\omega^{*}\right) \varphi\left(\theta^{*} ; \theta^{(g)}, \Psi\right)} \frac{\pi\left(\theta^{*}\right)}{\pi(\theta)} \\
& \quad=\frac{\mathrm{e}^{q\left(x ; \theta^{*}\right)} / c\left(\theta^{*}\right)}{\mathrm{e}^{q(x ; \theta)} / c(\theta)} \frac{P_{\zeta, \theta^{*}}^{B}\left(\omega^{*}\right)}{P_{\zeta, \theta}^{B}(\omega)} \frac{P_{\theta, \zeta}^{F}(\omega) \varphi\left(\theta^{(g)} ; \theta^{*}, \Psi\right)}{P_{\theta^{*}, \zeta}^{F}\left(\omega^{*}\right) \varphi\left(\theta^{*} ; \theta^{(g)}, \Psi\right)} \frac{\pi\left(\theta^{*}\right)}{\pi(\theta)} .
\end{aligned}
$$


Upon writing out the distributions $P_{\theta, \zeta}^{F}$ and $P_{\zeta, \theta}^{B}$, and rearranging, the acceptance ratio reduces to

$$
\frac{\mathrm{e}^{q\left(x ; \theta^{*}\right)}}{\mathrm{e}^{q(x ; \theta)}} \frac{Q_{\zeta, \theta^{*}}^{B}\left(\omega^{*}\right)}{Q_{\theta^{*}, \zeta}^{F}\left(\omega^{*}\right)} \frac{Q_{\theta, \zeta}^{F}(\omega) \varphi\left(\theta^{(g)} ; \theta^{*}, \Psi\right)}{Q_{\zeta, \theta}^{B}(\omega) \varphi\left(\theta^{*} ; \theta^{(g)}, \Psi\right)} \frac{\pi\left(\theta^{*}\right)}{\pi(\theta)},
$$

which only contains analytically tractable expressions. The details of how to construct $P_{\theta, \zeta}^{F}$, and $P_{\zeta, \theta}^{B}$, are described in [29], but the algorithm has an equivalent interpretation in terms of the linked importance sampler (LIS) of Neal [41]. The LIS is an importance sampler, where $\lambda(\theta, \zeta ; \omega)$ is an estimator of $\lambda(\theta, \zeta)=c(\zeta) / c(\theta)$, in the sense that $E_{P_{\theta, \zeta}^{F}}(\lambda(\theta, \zeta ; \omega))=\lambda(\theta, \zeta)$. Hence, running LISA amounts to proposing a move to $\theta^{*}$, estimating $\lambda\left(\theta^{*}, \zeta\right)$ using LIS, and accepting the move with an acceptance ratio that is equal to a ratio of normal densities times $\pi\left(\theta^{*} \mid x\right) / \pi\left(\theta^{(g)} \mid x\right)$ except that $\lambda\left(\theta^{*}, \theta^{(g)}\right)$ is substituted by $\lambda\left(\theta^{*}, \zeta ; \omega^{*}\right) / \lambda\left(\theta^{(g)}, \zeta ; \omega\right)$. The denominator in the latter expression is the estimate of $\lambda\left(\theta^{(g)}, \zeta\right)$, from when $\theta^{(g)}$ was accepted for the first time. The accuracy of $\lambda(\theta, \zeta ; \omega)$ and consequently the mixing of the MCMC depends on and can be tuned by adjusting the dimensions of the state space $\Omega$, through $K$ and $m$.

\subsection{The choice of $\zeta$ and $\Psi$}

The role of $\zeta$ is essentially to define a reference distribution against which the pmf defined by $\theta$ is evaluated. The simplest form of the auxiliary variable algorithm of Møller et al. [40] may be considered the special case of LISA when $K=m=1$. In this case $\Omega=\mathcal{X}, P_{\zeta, \theta}^{B}(\omega)=p(\omega \mid \zeta)$, and $P_{\theta, \zeta}^{F}(\omega)=p(\omega \mid \theta)$, with the interpretation that $\exp \{q(\omega ; \zeta)-q(\omega ; \theta)\}$ is used to estimate $\lambda(\theta, \zeta)$ using (one) $\omega$ drawn from the importance distribution $p(\omega \mid \theta)$. This illustrates why the choice of a "good" $\zeta$ is essential for the performance of LISA (for an example of adverse effects of less good choices see further [29]; Møller et al. [40] discuss the choice of $\zeta$ for the auxiliary variable MCMC at length and the accuracy of LIS as a function of the difference between $\zeta$ and $\theta$ is discussed in [41]).

With constant prior distributions a good choice of $\zeta$ is one that is equal to the modal point in the posterior distribution, i.e. the MLE. Assuming this to hold true also in the case of missing data, where $x=(u, v)$ will vary between iterations, $\zeta$ should be set to the maximiser of $\ell(\theta ; u)=\log L(\theta ; u)$. The gradient of the posterior with a constant prior is the differentiated log likelihood,

$$
\frac{\partial}{\partial \theta} \ell(\theta ; u)=\frac{\frac{\partial}{\partial \theta} \sum_{v} p(u, v \mid \theta)}{\sum_{v} p(u, v \mid \theta)}-\frac{\partial}{\partial \theta} \psi(\theta)
$$

which is the score function, $S(\theta ; u)$. In the general case when $q(x ; \theta)=\eta(\theta)^{\mathrm{T}} z(x)$,

$$
S(\theta ; u)=\dot{\eta}(\theta)^{\mathrm{T}}\left[E_{\eta(\theta)}(z(u, v) \mid u)-E_{\eta(\theta)}(z(x))\right],
$$

where $\dot{\eta}\left(\theta_{0}\right)=\left.((\partial / \partial \theta) \eta(\theta))^{\mathrm{T}}\right|_{\theta=\theta_{0}}$, and $E_{\eta}(\cdot \mid u)$ is the conditional expectancy on $\mathcal{X}_{M}(u)$. The Hessian equals

$$
\begin{aligned}
H(\theta) & =\frac{\partial}{\partial \theta} S(\theta ; u)^{\mathrm{T}} \\
& =\left[E_{\eta(\theta)}(z(u, v) \mid u)-E_{\eta(\theta)}(z(x))\right]^{\mathrm{T}} \ddot{\eta}(\theta)-\dot{\eta}(\theta)\{I(\eta)\} \dot{\eta}(\theta)^{\mathrm{T}},
\end{aligned}
$$

where $\dot{\eta}\left(\theta_{0}\right)=\left.\left(\left(\partial^{2} /\left[\partial \theta \partial \theta^{T}\right]\right) \eta(\theta)\right)^{\mathrm{T}}\right|_{\theta=\theta_{0}}$, and

$$
-I(\eta)=\left.\frac{\partial}{\partial \eta}\left[E_{\eta}(z(u, v) \mid u)-E_{\eta}(z(x))\right]\right|_{\theta=\theta_{0}}=\operatorname{Cov}_{\eta}(z(u, v) \mid u)-\operatorname{Cov}_{\eta}(z(x)) .
$$

For the regular exponential family case $H(\theta)$ simplifies to $-I(\theta)$, and $S(\theta ; u)=\left[E_{\theta}(z(u, v) \mid u)-\right.$ $E_{\theta}(z(x))$ ]. In this case, the MLE may be found using the Robbins-Monro procedure [50] or by approximating $L(\theta ; u)$ by its MCMC analogue as in [20]. For the curved exponential form, one may not make 
use of the standard likelihood equation to find the MLE. In the case where there are no missing data, an approximate Fisher scoring algorithm may be implemented as shown by $[26,16]$. In the case when we have missing data, we may find the modal point in the posterior distribution by iteratively updating according to $\zeta^{(t)}=\zeta^{(t-1)}-J\left(\zeta^{(t-1)}\right)^{-1} S\left(\zeta^{(t-1)} ; u\right)$, where $J(\zeta)=\dot{\eta}(\zeta)\{I(\eta)\} \dot{\eta}(\zeta)^{\mathrm{T}}$, and where the expectancies are approximated using importance samples. It is important to note however that for the purposes of implementing LISA we do not require the exact solution but only a $\zeta$ which is good enough. When approximating the expected value $E_{\eta}(z(x))$ and $\operatorname{Cov}_{\eta}(z(x))$, we may use the standard Metropolis-Hastings approach $[7,8,51,18]$. Approximating the conditional expectancies $E_{\eta}(z(u, v) \mid u)$ and $\operatorname{Cov}_{\eta}(z(u, v) \mid u)$ needs only the minor modification of only proposing to update elements $v$.

In addition to the choice of $\zeta$, the mixing of the chain $\left(\theta^{(g)}, \omega^{(g)}\right)$ depends on the choice of proposal distribution in standard fashion (see e.g. [6]). Settling on the choice of $N\left(\theta^{(g-1)}, \Psi\right)$ for $\theta^{*}$ in iterationg, the efficiency of the proposal density is affected by the choice of $\Psi$. Tierney [56], and Roberts, Gelman and Giles [43] show that $\Psi=c / \sqrt{1+p} \Sigma$ is an efficient choice, where $c>0$ is a constant and $\Sigma$ is the variance covariance matrix of the target distribution. For LISA with missing data, we use the inverse of the Fisher information matrix as an approximation of $\Sigma$ and allow $c$ to be rather lower than in the ideal case considered in [56]. The reason for the latter is the added uncertainty introduced by the need to also draw $\omega$ and $v$.

\subsection{Drawing from the full conditional distribution of the missing tie-variables}

An updating step in the Metropolis-Hasting algorithm for drawing $v$ used here is constructed as follows (for alternative updating steps see [51]). Assuming we wish to $\operatorname{draw} x \in \chi_{A}(u)$, for $A \subseteq \mathcal{N}$, and consequently only the elements in $A^{c}=\mathcal{N} \backslash A$ are allowed to change. Writing $\Delta_{e} x$ for the matrix that is equal to $x$ for all elements but has element $e$ set to $1-x_{e}$, we may propose to change the current state $x$ to $\Delta_{e} x$, where $e$ is chosen uniformly at random from among the elements of $A^{c}$, and accept this proposed move with a probability that is equal to the minimum of 1 and

$$
\frac{p\left(\Delta_{e} x \mid \theta\right)}{p(x \mid \theta)}=\exp \left\{q\left(\Delta_{e} x ; \theta\right)-q(x ; \theta)\right\} .
$$

A commonly used fact is that for ERGMs $q\left(\Delta_{e} x ; \theta\right)-q(x ; \theta)$ may be written in terms of the so-called change statistics, $\theta^{\mathrm{T}} \delta_{e} z(x)=\theta^{\mathrm{T}}\left(z\left(\Delta_{e} x\right)-z(x)\right)$, something which leads to considerable computational advantages $[11,7,8,51,18,52]$. Note that for the curved exponential family for fixed $\theta, \eta(\theta)$ and $z$ still constitute a non-curved ERGM, wherefore the ratio of pmf's may still be written in terms of the change statistics $\eta(\theta)^{\mathrm{T}} \delta z(x)=\eta(\theta)^{\mathrm{T}}\left(z\left(\Delta_{e} x\right)-z(x)\right)[24,26]$.

In the case of missing data, the chain $\left(\theta^{(g)}, \omega^{(g)}\right)$ is thus augmented by the tie variables $v_{1}, \ldots, v_{n_{v}}$, for $e_{1}, \ldots, e_{n_{v}} \in \mathcal{M}$. The updating step is: set $v_{k}^{(g)}=1-v_{k}^{(g-1)}$ with probability $\min (1, H)$ for

$$
H=\frac{p\left(1-v_{k}^{(g-1)} \mid \theta^{(g)}, \omega^{(g)}, u, v_{1}^{(g)}, \ldots, v_{k-1}^{(g)}, v_{k+1}^{(g-1)}, \ldots, v_{n_{v}}^{(g-1)}\right)}{p\left(v_{k}^{(g-1)} \mid \theta^{(g)}, \omega^{(g)}, u, v_{1}^{(g)}, \ldots, v_{k-1}^{(g)}, v_{k+1}^{(g-1)}, \ldots, v_{n_{v}}^{(g-1)}\right)} .
$$

Denoting the current state by $x$, the proposed move is to $\Delta_{e_{k}} x$, and the log acceptance ratio expressed in terms of change statistics is $\log H=\eta(\theta)^{\mathrm{T}} \delta_{e} z(x)$.

\subsubsection{Exploring the unobserved structure and link prediction}

From the properties of the MCMC algorithm it follows that marginally, $\left(v^{(g)}\right)_{g=1, \ldots, G}$ is a sample from the distribution $\pi(v \mid u)=\int_{\Theta} p(v \mid \theta, u) \pi(\theta \mid u) \mathrm{d} \theta$ (with the usual caveats regarding burn-in, thinning, etc.). Integrating out the parameter we effectively have a probability distribution for what is missing, $v$, given only what we have observed, $u$. We may ultimately be interested in studying some features that require information on the entire graph, either because these are graph level measures or simply because the measured properties would change with different configurations of $v$. If $v$ is missing we cannot calculate these measures (or they might be highly misleading) with anything less than filling in $v$. Since we have an entire distribution of $v$ 's at our disposal, we may nonetheless obtain the probabilities of different values of the measures given what we have observed.

The use of simulation to understand model behaviour [18] and making sense of the model has been a frequently employed tool [47]. Lately simulation from a fitted model has become a commonly 
employed diagnostics tool for assessing to what extent a model is able to capture important features of the data, $x$, that were not explicitly modelled [25]. Here instead we compare to $(u, v)$ over the distribution over $v$.

More formally, let $T(x)$ be some function of graphs $x \in \mathcal{X}$, such as for example betweenness [12] or constraint [4], then the pmf of $T$ given that we have only observed $u$ is given by $\pi(t \mid u)=$ $\operatorname{Pr}(T(u, v)=t \mid u)=\sum_{v: T(u, v)=t} \pi(v \mid u)$. From $\left(\theta^{(g)}, \omega^{(g)}, v^{(g)}\right)$, we may obtain a sample from the distribution $\pi(t \mid u)$, simply as $t^{(g)}=T\left(u, v^{(g)}\right)$ and from $\omega^{(g)}$, we obtain a sample from $\pi(x \mid u)$. The MCMC estimator of $\operatorname{Pr}(T(u, v) \in A \mid u)$ is straightforward to obtain as the relative frequency $\frac{1}{G} \sum 1\left\{T\left(u, v^{(g)}\right) \in A\right\}$. When feasible using Rao-Blackwellisation (cf [14,54]) is more efficient.

\subsection{Approximate Bayesian inference}

As the updating step of $\theta$ is time consuming, it would be useful to be able to approximate this step for routine applications for large data sets. Two main approaches suggest themselves: approximate the $\log$-ratio $\psi\left(\theta^{(g)}\right) / \psi\left(\theta^{*}\right)$ numerically using importance sampling (see [15], for a review), and, employ a series expansion of the posterior about its modal point. The first approach does however carry with it several drawbacks [40].

For constant prior, we may approximate the posterior by expanding the log likelihood around the modal point, giving the familiar approximation $\theta \mid u \sim N_{p}\left(\zeta, J(\zeta)^{-1}\right)$. For the curved form, we assume that the Fisher information matrix is sufficiently close to the negative Hessian.

This approximate algorithm is a straightforward and easy to implement way of drawing $\left(v^{(g)}\right)$. However, it is hard to see how the adequacy of the approximation for $\left(\theta^{(g)}\right)$ could be ascertained by anything other than applying an MCMC scheme like that of LISA (for examples of discrepancies between the approximation and the true posterior see [33]). For the example in Fig. 6 considered in Section 5 , the joint posterior of $\theta_{7}$ and $\theta_{8}$ in Fig. 8 appears to differ substantially in form from normal densities.

\section{Examples}

The application of the data augmentation scheme is illustrated and some of the above issues highlighted using the collaboration network of Lazega's [37] $n=36$ law firm partners and a model specification that frequently has been used for this data to illustrate various aspects of ERGMs [52, 26,20,24,57]. More specifically, this model has the statistics: edges $z_{1}(x)=\sum_{i<j} x_{i j}$; main effect of seniority $z_{2}(x)=\sum_{i<j} x_{i j}\left(\mathrm{SEN}_{i}+\mathrm{SEN}_{j}\right)$, SEN $i$ reversed rank order of seniority divided by $n$; main effect of practice $z_{3}(x)=\sum_{i<j} x_{i j}\left(\mathrm{PRA}_{i}+\mathrm{PRA}_{j}\right)$, $\mathrm{PRA}_{i}$ equal to one (zero) according to whether partner $i$ practices corporate law (litigation); homophily of practice $z_{4}(x)=\sum_{i<j} x_{i j} \mathbf{1}\left\{\mathrm{PRA}_{i}=\mathrm{PRA}_{j}\right\}$; homophily of sex $z_{5}(x)=\sum_{i<j} x_{i j} \mathbf{1}\left\{\mathrm{MAN}_{i}=\mathrm{MAN}_{j}\right\}$; homophily of office $z_{6}(x)=\sum_{i<j} x_{i j} \mathbf{1}\left\{\mathrm{OFF}_{i}=\right.$ $\left.\mathrm{OFF}_{j}\right\}$, for $\mathrm{OFF}_{i}$ being a categorical variable denoting whether $i$ works in the $\mathrm{A}, \mathrm{B}$ or $\mathrm{C}$ office.

In addition we assume the curved exponential form of Hunter and Handcock [26] whose simple ERGM projection has $z\left(x ; \theta_{p-1}\right)=\left(z_{1}(x), \ldots, z_{6}(x), A K T\left(x ; \mathrm{e}^{\theta_{p}}\right)\right)^{\mathrm{T}}$ as its vector of statistics, i.e. dyadic covariates one through six, and the alternating $k$-triangle statistic [52], or geometrically shared partners [26,24]. For the function $\eta(\theta)$ and associated vector of statistics, we refer to [26] and [24]. For numerical summaries of the model fitted to the complete network see Table 4 in [29]. In the following, we illustrate the effect of missing data in detail for a few extreme cases with missing actors and tievariables missing as a consequence of snowball sampling, after which we present a more extensive example with missing dyads.

\subsection{The effect of missing data}

\subsubsection{Actor-dependent missing data}

The effect of the missing data on inference may be viewed from the perspective of the posterior of $\theta$ and summary measures thereof, or the degree of structural information retained. Some regions of 
a

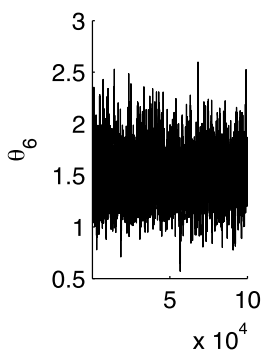

e

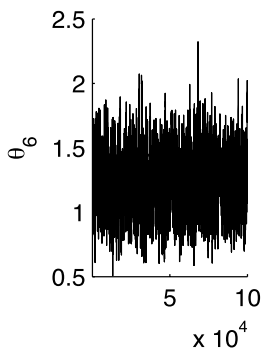

$\mathrm{b}$

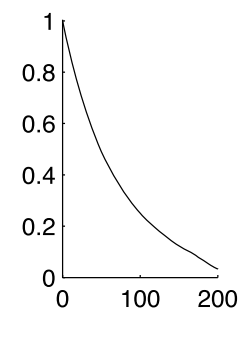

$f$

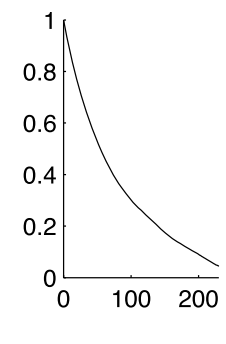

C

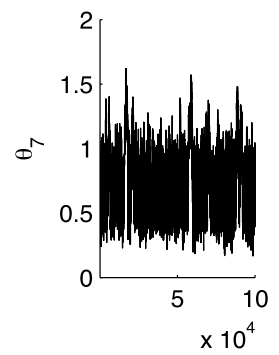

9

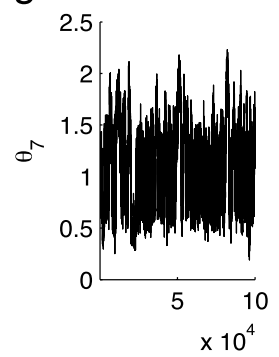

d

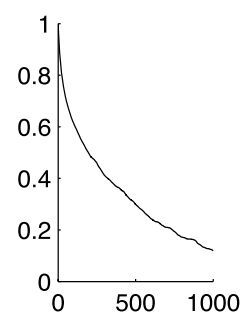

$\mathrm{h}$

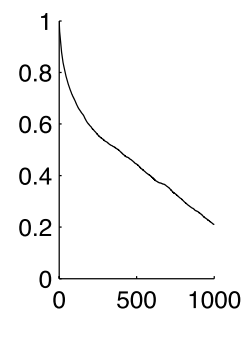

Fig. 1. Trace plots and sample autocorrelation functions $\theta_{6}$ (office homophily) and $\theta_{7}$ (edgewise shared partners) for nonrespondents; case 1 (a through d), and case 2 (e through $\mathrm{f}$ ).

the graph provide more information or at least different information than others. What exactly would constitute a region of the graph however, may not be straightforward to define but in the particular case of non-respondents, the regions may simply be couched in terms of the actors.

Clearly the tie-variables of some actors are going to have a greater impact on the inference than others. Koskinen, Wang, Robins and Pattison [31] propose a range of influence measures to investigate the role of different actors in the network from a model-based perspective. In the following, we remove one actor (case 1) that according to those criteria would result in the greatest change in fit. We also remove an actor (case 2) who has a very small influence on the model. All estimations are based on thinned samples of a total of 100,000 iterations with $K=7000$ and $m=9$, and $\zeta$ and $\Psi$ set as described in Section 3.2 (no data sets were degenerate and the algorithm for $\zeta$ always converged except in one case that required some further effort). Trace plots and autocorrelation functions for two parameters and cases 1 and 2 are given in Fig. 1. The homophily parameter mixes well (effective sample size, ESS, 908 for (panels a and b) and 602 (panels e and f)) the clustering, or edgewise shared partner parameter, less so (effective sample size, ESS, 145 for (panels c and d), and 109 (panels g and $\mathrm{h}$ )). A Gaussian distribution badly approximates the distribution of $\theta_{7}$ and $\theta_{8}$ (see e.g. Fig. 2 and the later Fig. 8), something which limits the ability to tune the proposal distribution (in this context, choosing $c$ ) to reduce autocorrelation while retaining a good acceptance rate.

That cases 1 and 2 represent two extremes is illustrated in the differences in posteriors Fig. 2 . The removal of an actor for case 1 has the most visible effect on $\theta_{6}$ (homophily office) and $\theta_{7}$ (alternating $k$-triangles). This mirrors the fact that the actor has many ties but is one of the few partners in the $\mathrm{C}$ office and sits in a highly triangulated zone, hence contributing greatly to the alternating $k$-triangle statistic. While the removed actor in case 2 has the well above average number of ties, his removal only adds to the uncertainty regarding $\theta_{7}$.

The differences between cases 1 and 2 in the posteriors for the $\theta_{k}$ 's are mirrored in the predictive probabilities for the tie-variables associated with the two actors (see Fig. 2(a)-(d)). The model does not manage to discriminate between the null-ties and actual ties in case 1-there is little difference between the Fig. 2(a) and (b). This not merely to do with the dyadic attributes as may be seen by 

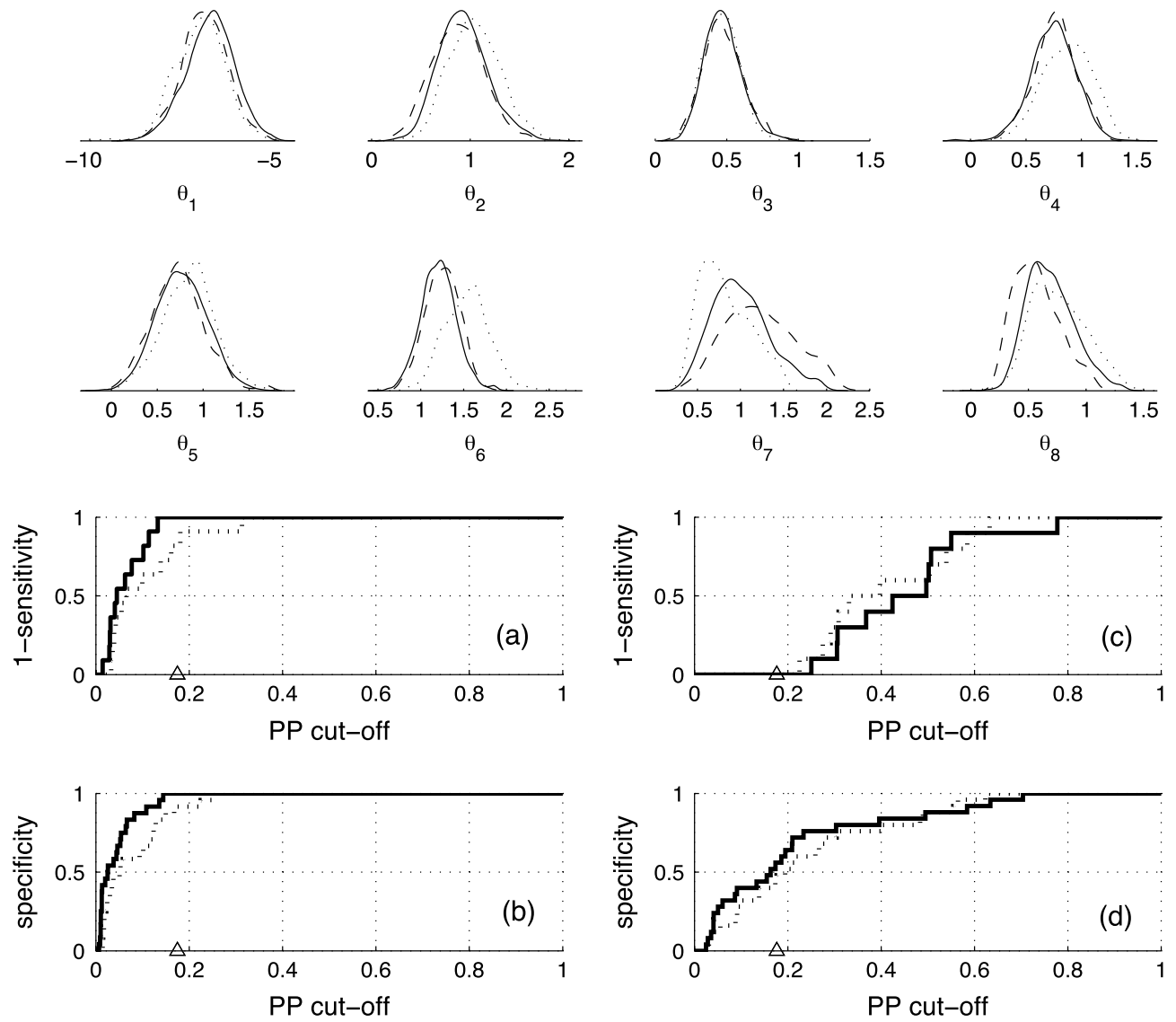

Fig. 2. Posteriors for $\theta$ (top 8 panels) for complete data (solid line), case 1 (dotted line), and case 2 (dashed line). Specificity and 1 - Sensitivity against posterior predictive (PP) cut-off value for case 1 (left) and case 2 (right) for full curved (solid), dyadindependent (dotted), and Bernoulli (triangle) models.

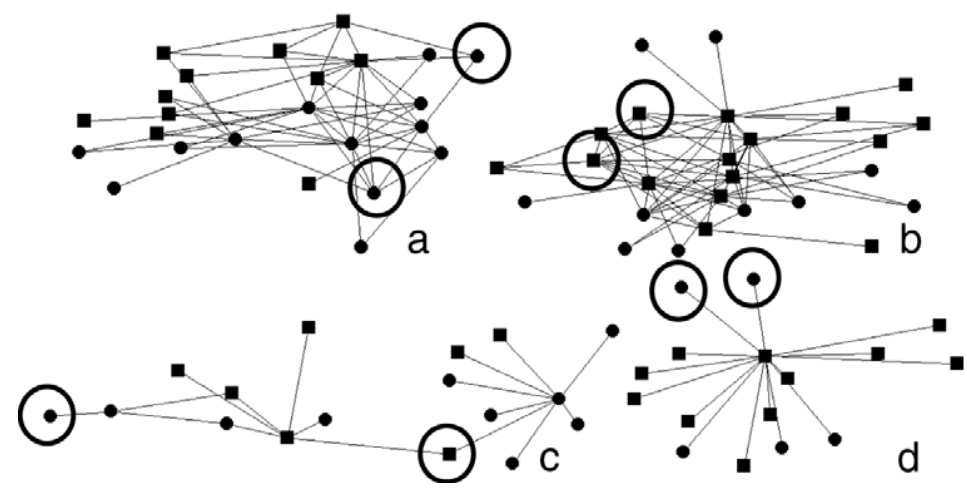

Fig. 3. Four inferentially degenerate snowball samples from Lazega's [37] 36 partners with seed nodes circled (litigation indicated by the box).

comparing the dyad-independent with the full model in Fig. 2). Furthermore, if the model containing only the edge parameter (indicated by a triangle in Fig. 2(a)-(d)) was to be used as reference for when a 

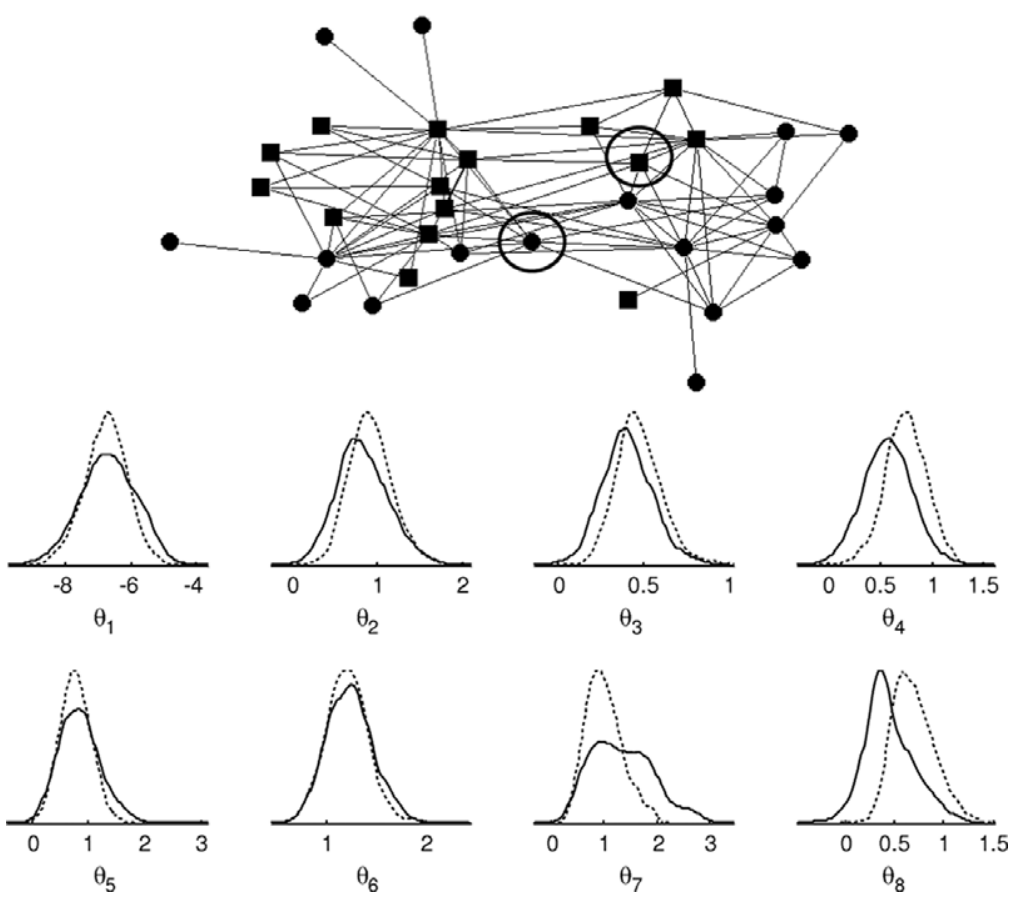

Fig. 4. Snowball sample from Lazega's [37] 36 partners with seed nodes circled and posterior distributions (true dashed line) for parameters.

tie is deemed to be present (predicted tie-probability greater than cut-off) or not (cut-off probabilities of 0.17 and 0.18 for cases 1 and 2 respectively), all null-ties of the missing actor in case 1 would be correctly classified but none of the present ties would be correctly classified, i.e. the specificity, or true negative rate (TNR), would be $100 \%$, and the sensitivity, or true positive rate (TPR), would be 0 . (If the posterior predictive probability that a missing tie-variable is equal to 1 is greater than the observed density, $|M|^{-1} \sum u_{e}$, then this is evidence for that the likelihood of the tie being present is greater than compared to the baseline probability of a tie). The dyad-independent model here does better for ties (TPR of 18\%) but worse for null-ties (TNR 92\%).

The tie-variables in case 2 are easier to predict; the full model has a TNR of $60 \%$ cent and a TPR of $100 \%$. Note here that the full model does a slightly better job of discriminating ties from null-ties than the dyad-independent model (that gives a TNR of $48 \%$ but it also underpredicts tie-probabilities for ties in the interval roughly between 0.2 and 0.5 ).

\subsubsection{Tie-dependent missing data}

Snowball sampling offers another aspect of degradation of the network information, in which the regions included and left out are dependent on the network structure itself. Here we consider snowball samples with a seed set of two nodes and two waves-the alters of ego and the alters of the alters (cf. Figure 1 in [20]). Snowball sampling was also used in [20] for illustrating the effect of missing data and is convenient since the sample is deterministic given the network and the seed set.

To illustrate the results in Section 2.1, consider what those results would imply for snowball samples taken on the Lazega [37] data set. In the following sociograms of samples, the actors not reported on are left out; however, since the population is known, the fact that $i$ is an alter of a seed node means that we have observed $x_{i k}$ for all $k \in V$.

The sample in Fig. 3(a) does not include any observed ties involving women and hence a model containing $\theta_{5}$ may not be estimated. The sample in Fig. 3 (b) captures 25 out of the 36 nodes and 330 out of the 630 dyads but main practice $\left(\theta_{3}\right)$ and homophily practice $\left(\theta_{4}\right)$ cannot be fitted at the same time. 

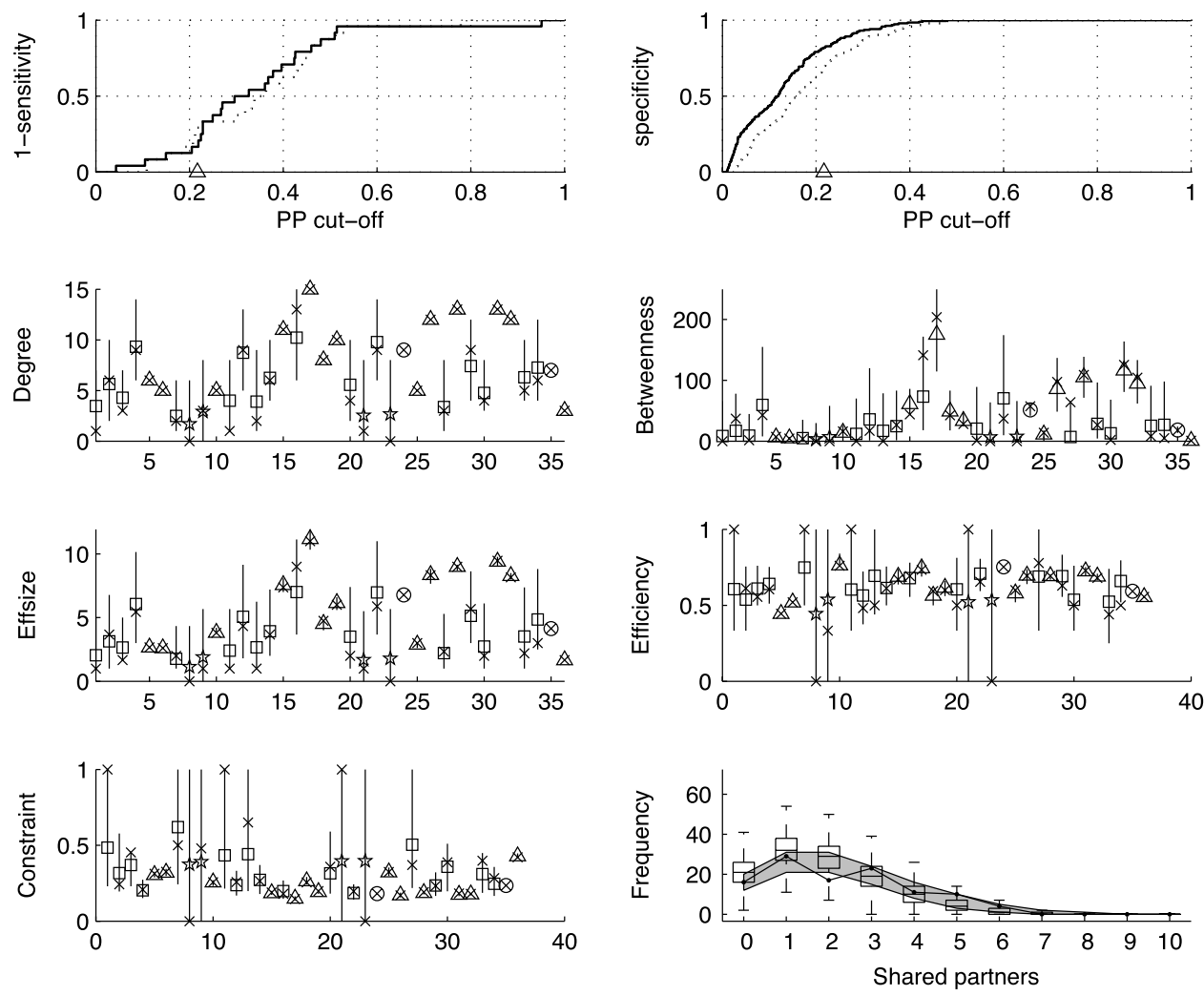

Fig. 5. Posterior predictive distributions for snowball sample in Fig. 4. Specificity and 1-Sensitivity against posterior predictive (PP) cut-off (top). For degree, betweenness and structural hole measures: 0.95 intervals (whiskers); true values (x); mean node in initial sample (circle); mean node in wave 1 (triangle); mean alters of wave 1 (square); mean rest (star). Posterior predictive shared partner distribution given snowball sample (boxes), completely observed data (shaded area), and observed complete data (connected points).

Samples Fig. 3(a) and (b) do not thus provide enough information to fit a dyad-independent model (referred to as "separation"; $[18,1,49]$ ) and by Observation 3 , the model that also includes $\theta_{7}$ and $\theta_{8}$ is inferentially degenerate.

For the sample in Fig. 3(c), the dyad-independent model may be fully estimated but the sample has no triangles and thus according to Proposition 4 and Observation 3, this sample is inferentially degenerate. The sample in Fig. 3 (d) has complete separation with respect to practice and does not have any triangles.

Here follow a couple of examples that allow inference, where in addition to prediction of ties, it might be interesting to look at characteristics of the graph that require increasingly detailed knowledge of the graph structure: the degrees of the nodes; betweenness [12,58]; and some structural hole measures [4].

The sample in Fig. 4 covers most of the network, 32 out of the 36 vertices are reported on and 420 out of the 630 dyads are observed. The marginal posterior distributions of the $\theta_{k}$ 's in Fig. 4 are very close to those for the complete data. Discriminating between ties and null-ties is relatively easy for the model. If we let the cut-off criterion for a tie be equal to the posterior predictive probability of the homogeneous Bernoulli model (triangle in top panels of Fig. 5), the TPR for the full model is $83 \%$, and $71 \%$ for the dyad-independent model; the TNR is $82 \%$ for the full model but only $68 \%$ for the dyad-independent model.

As all ties of seed nodes and their alters are observed, there is no uncertainty about their degrees (circles and triangles in of Fig. 5), but there is some uncertainty about the degrees of the rest of the 


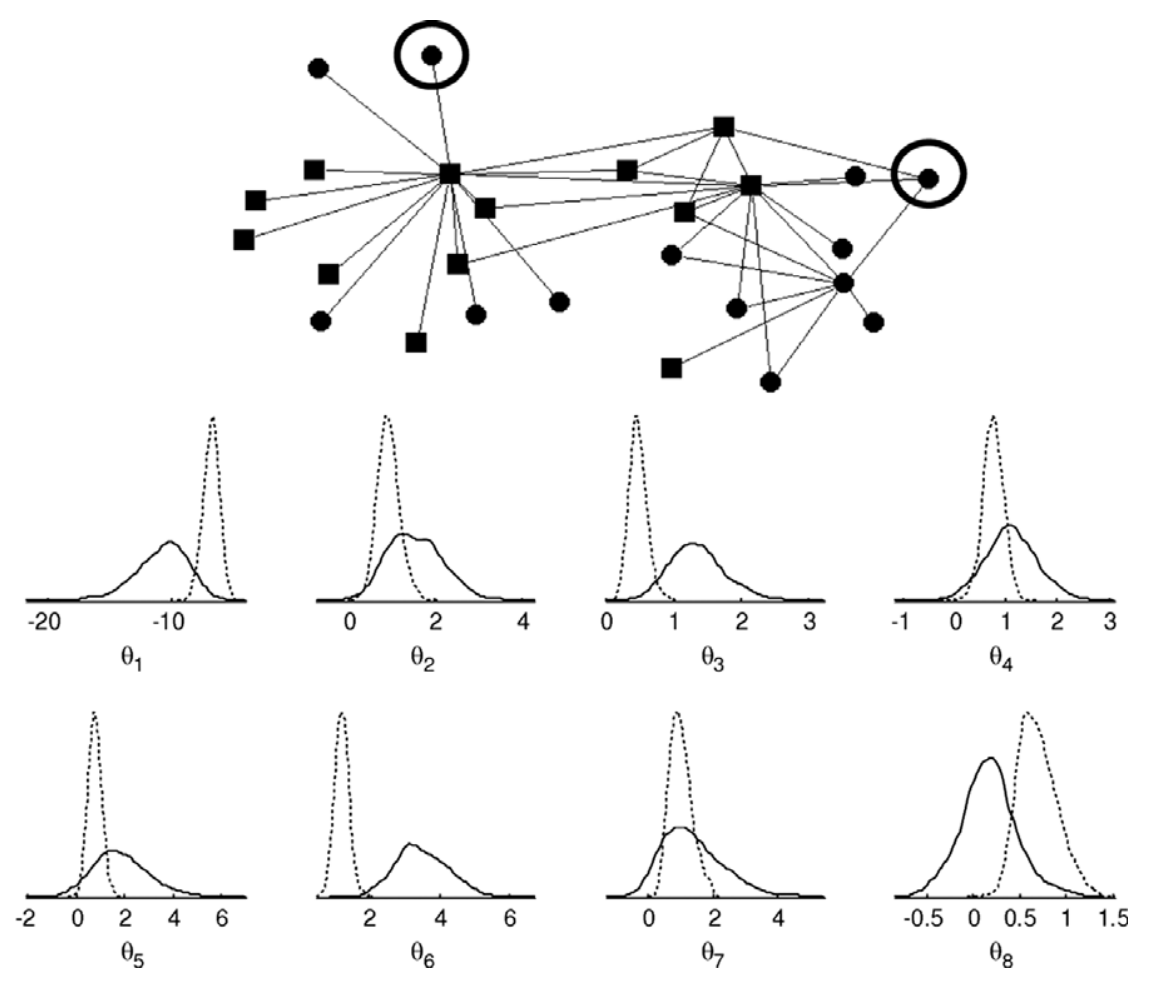

Fig. 6. Snowball sample from Lazega's [37] 36 partners with seed nodes circled and posterior distributions (true dashed line) for parameters.

nodes (as reflected in the prediction intervals in Fig. 5). For calculating the betweenness we need a little more information which is reflected in the fact that there is extra uncertainty as compared to the degrees in Fig. 5. As an example, while we have observed that node 17 has a degree of 15, we do not know whether 17 is on a geodesic [58] between 1 and 11 since the tie-variable $x_{1,11}$ is unobserved. On the whole, the true betweenness scores are well predicted by the model.

For the three structural hole indicators [4], Effsize (effective size) looks and performs like the predictions of the degrees, and efficiency (effective size divided by degree) and constraint perform well if we disregard the four non-sampled actors (the normalization makes efficiency and constraint perform poorly for the missing nodes as they all have low degrees or are isolated). The posterior predictive distribution for the number of shared partners has a little more uncertainty than for the completely observed data (out-liers not shown here) which stems from the greater uncertainty for $\theta_{7}$.

An illustrative way in which excluded regions affect inference is provided by using two seed nodes at opposite "ends" of the graph (Fig. 6), giving 26 out of the 36 actors observed and only 195 out of the 620 dyads observed. The graph has two main clusters in which all nodes belong to the same office and there are only five ties between the clusters. Consequently, the office homophily $\left(\theta_{6}\right)$ is over estimated and the non-homophilous ties of bridging nodes 32 and 31 are not predicted (Fig. 7). The hub nodes in each cluster practice litigation leading to overestimation of the corresponding main effect $\left(\theta_{3}\right.$; the betweenness of these two nodes is also overestimated, see Fig. 7). The clustering parameters (Fig. 8) and the shared partner distribution are affected by the left-hand cluster being dominated by a star and the right-hand cluster being more clustered. Using the same cut-off for the posterior predictive tie-probability the TPR is 65\% and the TNR 62\% for the full model (see Fig. 7), and for the dyad-independent model $68 \%$ and 59\%, for the TPR and TNR respectively. 

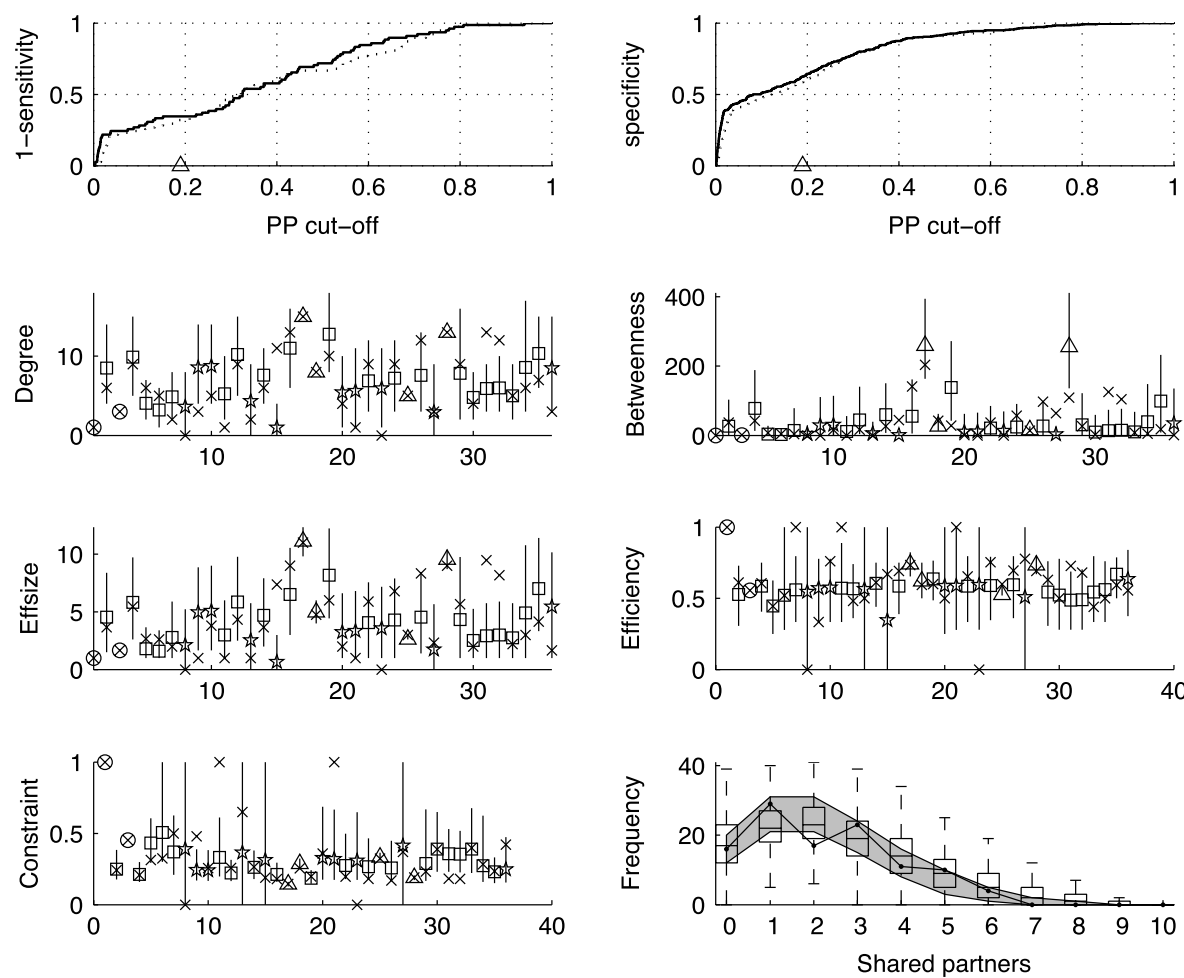

Fig. 7. Posterior predictive distributions for snowball sample from Lazega's [37] 36 partners with seed set in Fig. 6. Specificity and 1-Sensitivity against posterior predictive (PP) cut-off (top). For degree, betweenness and structural hole measures: 0.95 intervals (whiskers); true values (x); mean node in initial sample (circle); mean node in wave 1 (triangle); mean alters of wave 1 (square); mean rest (star). Posterior predictive shared partner distribution given snowball sample (boxes), completely observed data (shaded area), and observed complete data (connected points).

\subsection{Randomly removed dyads}

To give a more general idea of the performance of the ERGM in the presence of missing data we fit the ERGM using the Bayesian data augmentation algorithm for the cases where we have 50,100, 150, and 200 tie-variables missing at random for pairs of actors. For each sample fraction we do 20 replicates. The symmetrised Kullback-Leibler divergence $E\left\{\log \frac{\pi(\theta \mid u)}{\pi(\theta \mid u, v)} \mid u\right\}+E\left\{\log \frac{\pi(\theta \mid u, v)}{\pi(\theta \mid u)} \mid u, v\right\}$ may be approximated using path sampling [15], simulating from conditional distributions conditional on $u$, upon noting that the normalizing constant in the posteriors as well as in the likelihoods cancel. The divergence increases with the number of dyads that are removed, as can be seen in the left-hand panel of Fig. 9, and the divergence also seem to depend on the number of ties that are removed through the density parameter (the right-hand panel of Fig. 9; that some values are negative is due to the numerical approximation).

Hoff [21] proposes a model for networks and prediction of networks that may take dependences between tie-variables into account by latent variables. For the missing dyads, we fit a probit form of the model proposed in section 5 of [21], which has the linear predictor $\theta^{\mathrm{T}} z\left(x_{i j}\right)+w_{i} \Lambda w_{j}^{\mathrm{T}}$ (without the triangle statistic) for $x_{i j}$. The $w_{i}$ 's are the rows of an $n \times 2$ orthonormal matrix $W$ and $\Lambda$ is $2 \times 2$ diagonal matrix of real numbers. For drawing from the full conditional posterior of $W$ we used [22]. We refer to this model as EIGM in the sequel.

The specificitiy and sensitivity are stable over the different sample fractions. Using the observed density as posterior predictive cut-off the false positive rate (FPR) is around $25 \%$ for the ERGM and $30 \%$ for the EIGM; the sensitivity is around 76-81\% for the ERGM and 75\% for the EIGM (Fig. 10(a)). Hence, 


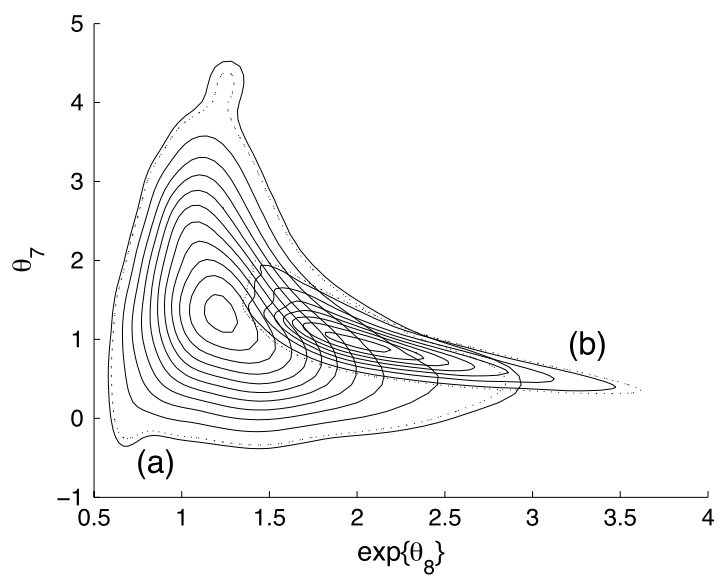

Fig. 8. Bivariate posterior distribution of clustering parameters compared for the complete data analysis (b) and missing data analysis based on snowball sample in Fig. 6 (a) 0.95 HPD regions indicated by the dashed line.
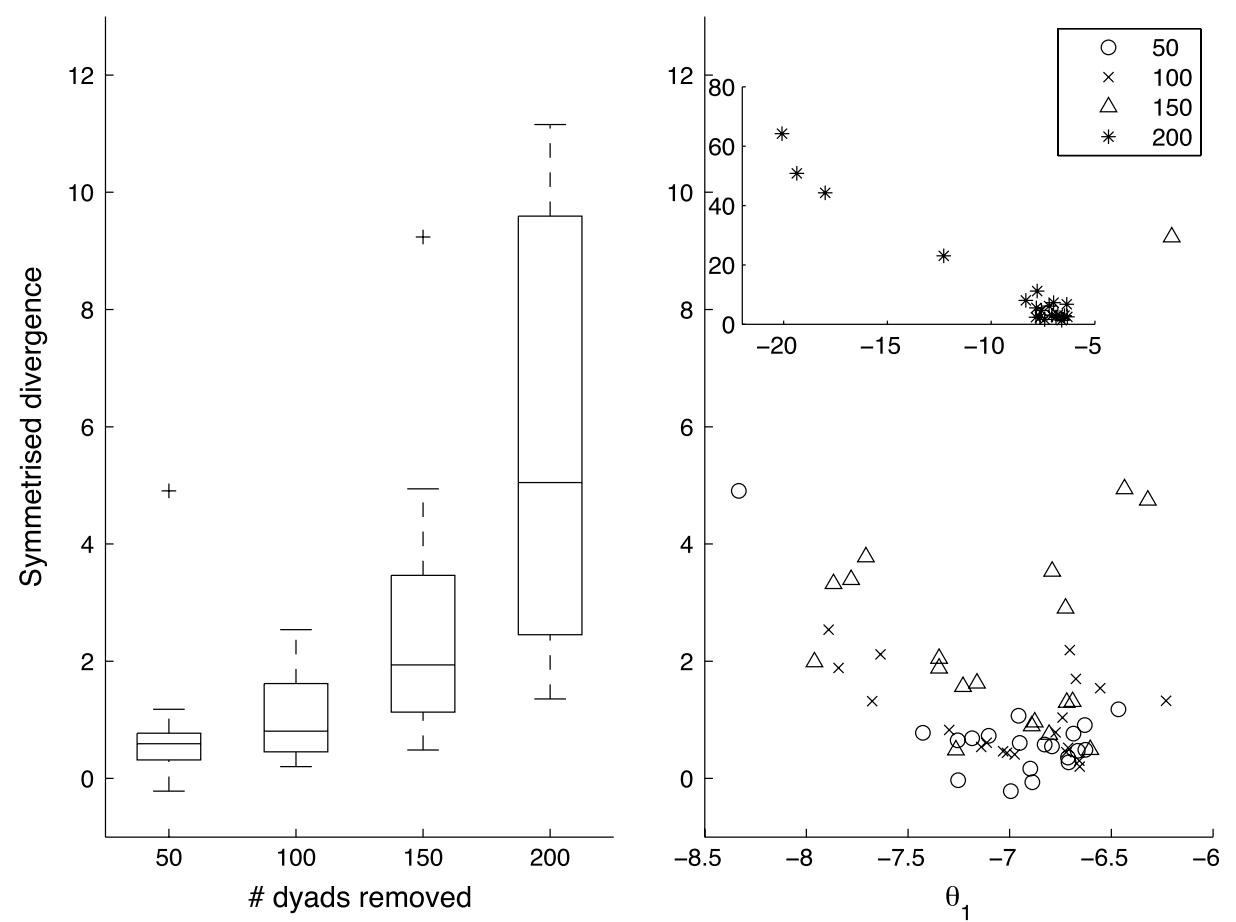

Fig. 9. The symmetrised Kullback-Leibler divergens (truncated at 13) between posteriors given $u$ and given ( $u, v$ ) for 20 replicates each for 50,100,150, and 200 dyads missing at random; symmetrised divergence against posterior mean of density parameter (200 missing dyads in insert).

using the observed density the EIGM overpredicts the number of ties slightly. When the predicted density $\left(\left|M^{c}\right|^{-1} \sum v_{e}\right)$ is used as the cut-off, the sensitivity is increased somewhat for the ERGM while the false negative rate (FPR, or 1 - TNR) is still lower than for the EIGM (around 26\% for the ERGM as compared to about 30\% for the EIGM). With the predicted density as the cut-off, the predictions of the ERGM outperforms those of the EIGM in the sense that the FPR is lower but the sensitivity is 

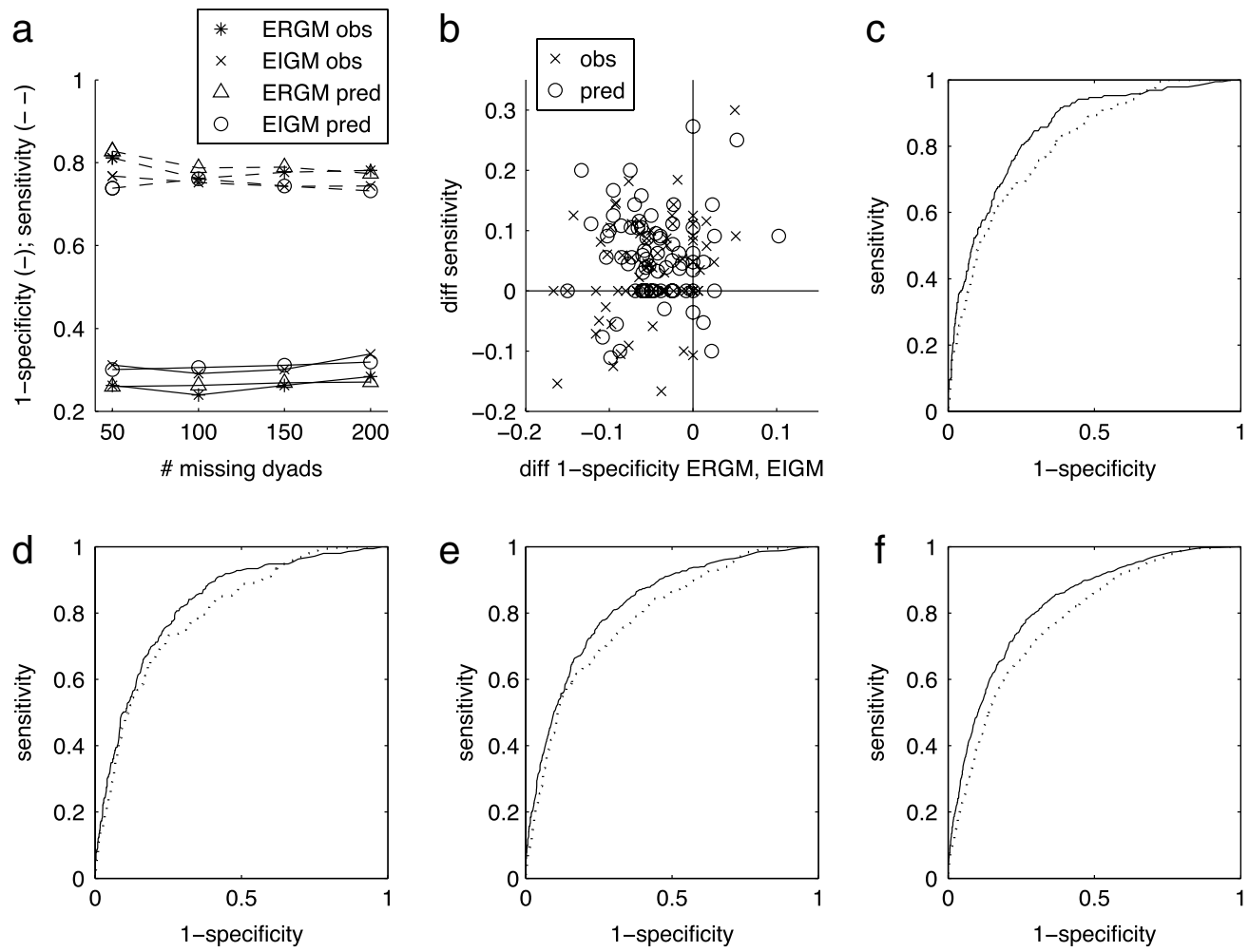

Fig. 10. Comparison of specificity and sensitivity for ERGM and the EIGM for 20 replicates each for 50, 100, 150, and 200 dyads missing at random. Average rates (a) and difference in rates (b) using observed (obs) density and predicted (pred) density as posterior predictive cutoff. ROC curves (ERGM, solid line; EIGM, dotted) combined for all replicates within missing fraction for 50 (c), 100 (d), 150 (e), 200 (f).

greater. Hence, a majority of replicates are in the upper-left quadrant of Fig. 10(b). Judging by the ROC curves, combined over replicates within sampling fractions, the ERGM provides a modest increase in performance over the EIGM.

\section{Conclusions and future directions}

We have presented and demonstrated the use of a Bayesian data augmentation scheme for fitting (curved) exponential family distribution models to social network data that have missing data. Although it is hard to characterise the nature of missing data and their effect on inference in general, we have demonstrated the consequence of missing data in a few fairly clear-cut examples. We have also provided some results regarding how much data may be degraded. In particular, we have provided an easy to check criterion for edges and alternating triangles model and also shown that models that have degenerate models nested in them are also degenerate. For a standard data set, the Bayesian tie prediction using ERGM performs at least as good as a competing model and manages to predict on average $80 \%$ of the ties correctly, allowing for $25 \%$ false positives with a third of data missing.

Future work include allowing for missing covariates (some results for missing covariates are presented in [30,32]) and link prediction. When link prediction is the primary aim it would be useful to relax the MAR assumption (Huisman [27] defines several "not MAR" missing data mechanisms) as well as incorporate prior information. In many instances "'not Mar" may be couched in terms of measurement error (as discussed in [32]). The choice of priors is not straightforward and is the topic of ongoing research. 
The approach, as we have seen, may be applied for snowball-sampled data, but its application to samples from large populations with an unknown total number of nodes presents some challenges. For large population sizes, the approximate procedure may prove useful, and it is is plausible that the homogeneity assumption has to be relaxed (as in [30]). An additional issue is the scaling properties of the ERGMs and [32] illustrate the consequences for inference of varying population size in snowball sampling.

\section{References}

[1] A. Albert, J.A. Anderson, On the existence of maximum likelihood estimates in logistic regression, Biometrika 71 (1984) $1-10$.

[2] Y.F. Atchade, N. Lartillot, C. Robert, Bayesian computation for statistical models with intractable normalizing constants, 2008, unpublished manuscript (available from http://www.stat.lsa.umich.edu/ yvesa/ncmcmc2.pdf).

[3] O.E. Barndorff-Nielsen, Information and Exponential Families in Statistical Theory, Wiley, New York, 1978.

[4] R.S. Burt, Structural Holes: The Social Structure of Competition, Harvard University Press, Cambridge, MA, 1992.

[5] R.S. Burt, A note on missing social network data in the general social survey, Social Networks 9 (1987) 63-73.

[6] S. Chib, E. Greenberg, Understanding the Metropolis algorithm, The American Statistician 49 (1995) 327-335.

[7] J. Corander, K. Dahmström, P. Dahmström, Maximum likelihood estimation for Markov graphs, Research Report, 1998:8, Stockholm University, Department of Statistics, 1998.

[8] J. Corander, K. Dahmström, R. Dahmström, Maximum likelihood estimation for exponential random graph model, in: J. Hagberg (Ed.), Contributions to Social Network Analysis, Information Theory, and Other Topics in Statistics; A Festschrift in honour of Ove Frank, Department of Statistics, University of Stockholm, 2002, pp. 1-17.

[9] E. Costenbader, T.W. Valente, The stability of centrality measures when networks are sampled, Social Networks 25 (2003) 283-307.

[10] P. Diaconis, D. Ylvisaker, Conjugate priors for exponential families, The Annals of Statistics 7 (1979) 269-281.

[11] O. Frank, D. Strauss, Markov Graphs, Journal of the American Statistical Association 81 (1986) 832-842.

[12] L.C. Freeman, A set of measures of centrality based on betweenness, Sociometry 40 (1977) 35-41.

[13] S.E. Fienberg, S. Wasserman, Categorical data analysis of single sociometric relations, in: S. Leinhardt (Ed.), Sociological Methodology, Jossey-Bass, San Francisco, 1981, pp. 156-192.

[14] A.E. Gelfand, A.F.M. Smith, Sampling based approaches to calculating marginal densities, Journal of the American Statistical Association 85 (1990) 398-409.

[15] A. Gelman, X.L. Meng, Simulating normalizing constants: From importance sampling to bridge sampling to path sampling, Statistical Science $13(1998)$ 163-185.

[16] C.J. Geyer, E. Thompson, Constrained Monte Carlo maximum likelihood for dependent data, Journal of the Royal Statistical Society Series B 54 (1992) 657-699.

[17] K. Gile, M.S. Handcock, Model-based Assessment of the Impact of Missing Data on Inference for Networks, Working Paper no. 66, Center for Statistics and the Social Sciences, University of Washington, 2006.

[18] M.S. Handcock, Assessing degeneracy in statistical models of social networks, Working Paper no. 39, Center for Statistics and the Social Sciences, University of Washington, 2003 (available from http://www.csss.washington.edu/Papers/wp39.pdf).

[19] M. Handcock, K. Gile, Modeling networks from sampled data, Annals of The American Statistician (2009) (forthcoming).

[20] M. Handcock, K. Gile, Modeling social networks with sampled or missing data, CSSS Working Paper 75, University of Washington, 2007 (available from http://www.csss.washington.edu/Papers/wp75.pdf).

[21] P. Hoff, Multiplicative latent factor models for description and prediction of social networks, Computational \& Mathematical Organization Theory (2008) doi:10.1007/s10588-008-9040-4.

[22] P. Hoff, Simulation of the Matrix Bingham-von Mises-Fisher distribution with applications to multivariate and relational data, Journal of Computational \& Graphical Statistics 18 (2009) 438-456.

[23] P. Holland, S. Leinhardt, An exponential family of probability distributions for directed graphs (with discussion), Journal of the American Statistical Association 76 (1981) 33-65.

[24] D.R. Hunter, Curved exponential family models for social networks, Social Networks 29 (2007) 216-230.

[25] D.R. Hunter, S.M. Goodreau, M.S. Handcock, Goodness of fit of social network models, Journal of the American Statistical Association 103 (2008) 248-258.

[26] D.R. Hunter, M.S. Handcock, Inference in curved exponential family models for networks, Journal of Computational and Graphical Statistics 15 (2006) 565-583.

[27] M. Huisman, Imputation of missing network data: Some simple procedures, Journal of Social Structure 10 (1) (2009).

[28] G. Kossinets, Effects of missing data in networks, Social Networks 28 (2006) 247-268.

[29] J.H. Koskinen, The linked importance sampler auxiliary variable metropolis hastings algorithm for distributions with intractable normalising constants, MelNet Social Networks Laboratory Technical Report 08-01, Department of Psychology, School of Behavioural Science, University of Melbourne, Australia, 2008 (available from http://www.sna.unimelb.edu.au/publications/MelNet_Techreport_08_01).

[30] J.H. Koskinen, Using latent variables to account for heterogeneity in exponential family random graph models, in: Proceedings of the 6th St. Petersburg Workshop on Simulation, 2009, pp. 845-849.

[31] J.H. Koskinen, P. Wang, G.L. Robins, P.E. Pattison, Extreme actors-Outliers and influential observations in exponential random graph (p-star) models, MelNet Social Networks Laboratory Technical Report 08-05, Department of Psychology, School of Behavioural Science, University of Melbourne, Australia, 2008 (available from http://www.sna.unimelb.edu.au/publications/MelNet_Techreport_08_05.pdf). 
[32] J.H. Koskinen, G.L. Robins, P.E. Pattison, Missing data in social networks: Problems and prospects for model-based inference, MelNet Social Networks Laboratory Technical Report 08-03, Department of Psychology, School of Behavioural Science, University of Melbourne, Australia, 2009.

[33] J.H. Koskinen, P. Wang, D.S. Lusher, G.L. Robins, Approximate and exact Bayesian goodness of fit for ERGMs ( $\left.p^{*}\right)$ in: Sunbelt XXVIII, Sunbelt Social Network Conference, Tradewinds Resort Hotel, St. Pete Beach, FL, January, 2008 (Paper presented).

[34] D. Krackhardt, Cognitive social structures, Social Networks 9 (1987) 109-134.

[35] E.O. Laumann, P.V. Marsden, D. Prensky, The boundary specification problem in network analysis, in: R.S. Burt, M.J. Minor (Eds.), Applied Network Analysis, Sage Publications, London, 1983, pp. 18-34.

[36] S.L. Lauritzen, Graphical Models, Oxford University Press, Oxford, 1996.

[37] E. Lazega, The Collegial Phenomenon: The Social Mechanisms of Cooperation Among Peers in a Corporate Law Partnership, Oxford University Press, Oxford, 2001.

[38] R.J.A. Little, D.B. Rubin, Statistical Analysis with Missing Data, John Wiley, New York, 1987.

[39] I. Murray, Z. Ghahramani, D.J.C. MacKay, MCMC for doubly-intractable distributions, in: Proceedings of the 22nd Annual Conference on Uncertainty in Artificial Intelligence (UAI), 2006.

[40] J. Møller, A.N. Pettitt, K.K. Berthelsen, R.W. Reeves, An efficient Markov chain Monte Carlo method for distributions with intractable normalising constants, Biometrika 93 (2006) 451-458.

[41] R.M. Neal, Estimating ratios of normalizing constants using linked importance sampling, in: Technical Report No. 0511, Department of Statistics, University of Toronto 2005 (available from http://arxiv.org/abs/math.ST/0511216).

[42] P.E. Pattison, S. Wasserman, Logit models and logistic regressions for Social Networks: II. multivariate relations, British Journal of Mathematical and Statistical Psychology 52 (1999) 169-193.

[43] G.O Roberts, A. Gelman, W.R. Gilks, Weak convergence and optimal scaling of random walk Metropolis algorithms, Annals of Applied Probability 7 (1) (1997) 110-120.

[44] G.L. Robins, P.E. Pattison, P. Elliot, Network models for social influence processes, Psychometrika 66 (2001) 161-190.

[45] G.L. Robins, P.E. Pattison, S. Wasserman, Logit models and logistic regressions for social networks, III. Valued relations, Psychometrika 64 (1999) 371-394.

[46] G.L. Robins, P.E. Pattison, J. Woolcock, Missing data in networks: exponential random graph ( $\mathrm{p}^{*}$ ) models for networks with non-respondents, Social Networks 26 (2004) 257-283.

[47] G.L. Robins, P.E. Pattison, J. Woolcock, Small and other worlds: Global network structures from local processes, American Journal of Sociology 110 (2005) 894-936.

[48] D.B. Rubin, Inference and missing data (with discussion), Biometrika 63 (1976) 581-592.

[49] T.J. Santner, D.E. Duffy, A Note on A. Albert and J. A. Andersons conditions for the existence of maximum likelihood estimates in logistic regression models, Biometrika 73 (1986) 755-758.

[50] T.A.B. Snijders, The Robbins-Monro algorithm for estimation of ERGMs with missing or sampled data, Working Paper, Nuffield College, University of Oxford 2007.

[51] T.A.B. Snijders, Markov chain Monte Carlo estimation of exponential random graph models, Journal of Social Structure 3 (2) (2002).

[52] T.A.B. Snijders, P.E. Pattison, G.L. Robins, M.S. Handcock, New specifications for exponential random graph models, Sociological Methodology 36 (2006) 99-153.

[53] D. Stork, W.D. Richards, Nonrespondents in communication network studies: Problems and possibilities, Group and Organization Management 17 (1992) 193-209.

[54] M.A. Tanner, W.H. Wong, The calculation of posterior distributions by data augmentation (with discussion), Journal of the American Statistical Association 82 (1987) 528-550.

[55] S.K. Thompson, O. Frank, Model-based estimation with link-tracing sampling designs, Survey Methodology 26 (2000) 87-98.

[56] L. Tierney, Markov chains for exploring posterior distributions (with discussion and a rejoinder by the author), The Annals of Statistics 22 (1994) 1701-1762.

[57] M. van Duijn, K. Gile, M.S. Handcock, A framework for the comparison of maximum pseudo-likelihood and maximum likelihood estimation of exponential family random graph models, Social Networks 31 (2009) 52-62.

[58] S. Wasserman, K. Faust, Social Network Analysis: Methods and Applications, Cambridge University Press, Cambridge, 1994.

[59] S. Wasserman, P.E. Pattison, Logit models and logistic regressions for social networks: I. An introduction to Markov graphs and $\mathrm{p}^{*}$, Psychometrika 61 (1996) 401-425. 